\title{
Provision of Nutrients to Young Santalum album L. by Different Host Species
}

\author{
K.P. Millaniyage ${ }^{1 *}$, S.M.C.U.P. Subasinghe ${ }^{1}$, D.S. Hettiarachchi ${ }^{2}$ \\ ${ }^{1}$ Department of Forestry and Environmental Science, University of Sri Jayewardenepura, Sri Lanka \\ ${ }^{2}$ Wescorp Group of Companies, 26, Coulson Way, Canning Vale, WA 6155, Australia \\ *kulunipiumika@gmail.com
}

\begin{abstract}
Due to the increasing demand and market price established for its valuable fragrant essential oil produced in the heartwood, Santalum album is subjected to over-exploitation for a long period in Sri Lanka. Since it is a hemi-parasitic tree which has the ability of absorbing certain nutrients from other host tree species via root connections, a comprehensive understanding of S. album-host relationship is of major importance in both commercial and conservation perspectives.
\end{abstract}

Therefore, the present study was conducted to determine the ability of supplying nitrogen, phosphorous, potassium and magnesium to young $S$. album plants by different host species. A S. album-host trial plantation located in Balangoda of Rathnapura District belonging to WM3 agro-ecological zone was used for this purpose. This trial consisted of 20 different combinations of two hosts of Sesbenia grandiflora, Gliricidia sepium, Grevillea robusta, Calliandra calothyrus, Erythrina variegate, Acacia auriculiformis and Coffea arabica per $S$. album. Leaf samples from $S$. album plants were collected for five times, from November 2012 to October 2014. Total nitrogen content was determined using Micro-Kjeldhal method while the potassium and magnesium levels were determined using atomic absorption spectroscopy for the collected leaves. Phosphorous content was analysed by colourimetric determination using a spectrophotometer.

According to the results, G. sepium/S. grandiflora host combination provided the highest nitrogen content. Two G. robusta plants provided the highest potassium level while the host combination of G. sepium/G. robusta provided the highest phosphorous content for S. album. The highest magnesium content was observed in the combination with two G. sepium plants. However, One-way ANOVA did not indicate any significant difference for the tested nutrients in $S$. album leaves.

Keywords: Santalum album, Host species, Leaf nutrients, Haustorial connections 